\title{
Theoretical Analysis of Cavitation in Inducers with Unequally Spaced Blades*
}

\author{
Hironori HORIGUCHI ${ }^{* *}$, Tomokazu TAKASHINA*** and Yoshinobu TSUJIMOTO****
}

The present paper describes about the effects of the inequality of blade spacing on the steady cavitation and its stability. The development process of the cavity in the cascade with unequally spaced blades is more complex in comparison with that in the cascade with equally spaced blades. The development process can be reasonably explained by the interaction between the local flow near cavity trailing edge and the leading edge of adjacent blade. The minimum cavitation number of the region of stable cavitation can be decreased by unequalizing the blade spacing. Various unstable cavitation appears in low cavitation number where the cavity is longer than the $65 \%$ of blade spacing.

Key Words: Inducer, Cavitation, Stability, Singularity Method, Blade Spacing

\section{Introduction}

In 4-bladed inducers, the alternate blade cavitation, the rotating cavitation and the asymmetric cavitation occur as the flow rate decreases before the head breaks down ${ }^{(1)}$. In 3-bladed inducers the rotating cavitation occurs at higher cavitation number as the alternate blade cavitation does not occur. This is one of the reasons why 4bladed inducer is used in Arian V.

Authors have tried to prevent the rotating cavitation due to the alternate blade cavitation induced by alternate cutback of the leading edge of blades in 4-bladed inducer experimentally and theoretically ${ }^{(2)-(4)}$. In a series of these studies, it was found that the region of rotating cavitation could be decreased by enhancing the alternate blade cavitation through cutting back the leading edge alternately.

Unequal spacing can also be used to enhance the alternate blade cavitation. In the present study, we tried to suppress the rotating cavitation by encouraging the occurrence of alternate blade cavitation by way of unequaliz-

* Received 7th December, 2005 (No. 04-1245). Japanese Original: Trans. Jpn. Soc. Mech. Eng., Vol.71, No.705, B (2005), pp.1247-1255 (Received 19th November, 2004)

** Graduate School of Engineering Science, Osaka University, 1-3 Machikaneyama, Toyonaka, Osaka 560-8531, Japan. E-mail: horiguti@me.es.osaka-u.ac.jp

*** Altech Corporation, 3-6-35 Nishiki, Naka-ku, Nagoya, Aichi 460-0003, Japan

**** Graduate School of Engineering Science, Osaka University, 1-3 Machikaneyama, Toyonaka, Osaka 560-8531, Japan ing the blade spacing alternately. Numerical calculations based on singularity method were used for this purpose.

Steady cavitation in the cascade with alternately unequally spaced blades and its stability are discussed. The effect of the inequality of blade spacing for the suppression of cavitation instabilities such as rotating cavitation are also discussed by comparing the results with the cavitation in the cascade with equally spaced blades.

\section{Nomenclature}

$A$ : Coefficient matrix

$B$ : Constant vector

$C$ : Length of blade chord

$f:$ Function defined in Eq. (3)

$h:$ Mean spacing

$i$ : Imaginary unit in space, $i^{2}=-1$

$j$ : Imaginary unit in time, $j^{2}=-1$

$k$ : Complex propagation velocity ratio in a frame moving with a cascade $=k_{R}+j k_{I}=\frac{\omega /(2 \pi)}{U_{T} /\left(Z_{N} h\right)}$

$k_{R}$ : Propagation velocity ratio in a frame moving with a cascade

$k_{I}$ : Decay ratio

$L$ : Distance between the leading edge of blade and the space with constant total pressure, $1000 C$ (Inlet duct length toward $x$-axis)

$L 0, L 1$ : Distance between the leading edge of blade and the throat of blade passage

$l$ : Cavity length

$N$ : Amplitude of axial velocity fluctuation at $\xi=-L$

$N_{B}, N_{C}$ : Number of discrete points on $s$-coordinate 
$n:$ Blade index

$P$ : Constant represents the ratio of the displacement of leading edge of blade to the mean spacing $h$. This constant is called the ratio of inequality in the present study

$p_{-L}:$ Pressure at $\xi=-L$

$p_{t}:$ Total pressure at $\xi=-L$

$p_{v}:$ Vapor pressure

$q$ : Strength of source

$R$ : Ratio of the displacement of leading edge of blade to the mean spacing $h$

$S$ : Position of discrete points on $s$-coordinate

$s$ : Coordinate stretching with fluctuating cavity length

$t$ : Time

$U$ : Velocity of main flow

$U_{T}$ : Peripheral velocity of cascade $=U \sin (\alpha+\beta)$

$u, v: x$ and $y$ components of velocity

$w$ : Complex velocity

$Z_{N}$ : Number of blade

$z:$ Complex plane $=x+i y$

$\alpha:$ Angle of attack

$\alpha^{\prime}$ : Local angle of attack

$\beta$ : Stagger angle

$\gamma:$ Strength of vortex

$\theta_{m, n}$ : Phase difference of the cavity length fluctuation in $n$th blade relative to that in $m$ th blade

$\rho:$ Density of fluid

$\sigma:$ Cavitation number $=2\left(p_{-L}-p_{v}\right) /\left(\rho U_{T}^{2}\right)$

$\omega:$ Complex angular velocity $=\omega_{R}+j \omega_{I}$

$\omega_{R}:$ Angular velocity

$\omega_{I}$ : Decay ratio

$\xi$ : Coordinate along the blade with the origin at the leading edge of blade

$\zeta$ : Coordinate parallel to the cascade with the origin on $x$-axis

Superscript

$\sim$ : Unsteady component

Subscripts

1, 2 : Cavity region and non-cavity region

$c$ : Cavity surface

$k:$ Index of discrete point

$n:$ Blade index

$s:$ Steady component

$t$ : Free vortex

\section{Outline of Analysis}

Flow model and analytical method are the same as those in Ref. (5) except that the blade spacing is unequal alternately. A brief explanation is given about the flow model and the analytical method in the present paper.

We consider a cascade of flat plates with chord length $C$ and stagger angle $\beta$ as shown in Fig. 1, with a mean flow of magnitude $U$ and an angle of attack $\alpha$ at upstream

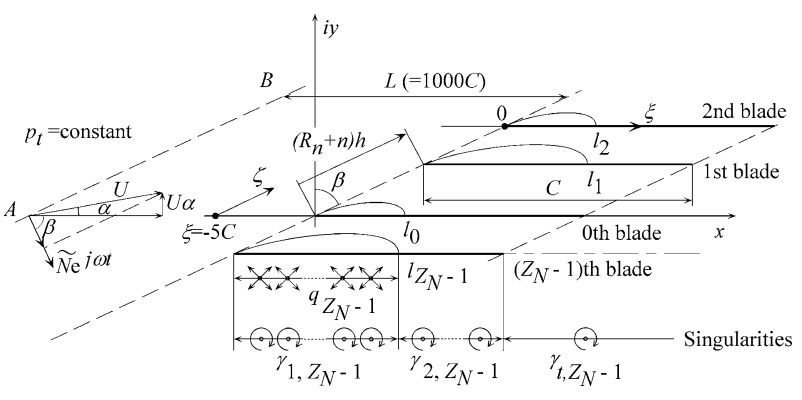

Fig. 1 Model for present analysis

boundary, $\xi=-L$. The complex velocity there is denoted by $\tilde{N} e^{i \beta} e^{j \omega t}$, where $\tilde{N}$ is an amplitude of fluctuating axial velocity, $i$ and $j$ denote the imaginary unit in space and time, respectively, and $\omega=\omega_{R}+j \omega_{I}$ is a complex frequency with its real part $\omega_{R}$ signifying the frequency and the imaginary part $\omega_{I}$ the decay ratio. The index $n$ of blade is defined by taking account of the periodicity of blade row for an inducer with the total blade number $Z_{N}$ : the blade located on $x$-axis is given the index zero, and the index increases in positive direction of $y$-axis and the index of $Z_{N}$ th blade returns to zero. Partial cavitation with cavity length $l_{n}$ on $n$th blade is considered.

In the present study, $Z_{N}$ is set to be 4 . To express the cascade with unequally spaced blades, the ratios $R_{n}(n=0$, $1,2,3)$ of the displacement of leading edge of blade to a mean spacing $h$ are introduced. $R_{n}$ are included in the term $\left(R_{n}+n\right) h$ in Fig. 1 and defined by

$$
\left\{\begin{array}{l}
R_{0}=R_{2}=0 \\
R_{1}=R_{3}=P
\end{array} .\right.
$$

where $P$ is a positive constant and called the ratio of inequality in the present study. By Eq. (1), the cascade with alternately unequally spaced blades can be expressed, whose blade spacing are $(1+P) h$ between 0 th $(2$ nd) and 1 st (3rd) blades and $(1-P) h$ between 1 st (3rd) and 2 nd (0th) blades.

The velocity disturbances due to the blades and cavities are represented as follows by source distributions $q_{n}$ on the cavity region, vortex distributions $\gamma_{1 n}$ and $\gamma_{2 n}$ on the blade, and trailing vortices $\gamma_{t n}$ on the wake surface of the blades:

$$
\begin{aligned}
w(z)= & u-i v=U e^{-i \alpha}+\tilde{N} e^{i \beta} e^{j \omega t} \\
& +\frac{1}{2 \pi} \sum_{n=0}^{Z_{N}-1}\left[\int_{0}^{1}\left\{q_{n}\left(s_{1}\right)+i \gamma_{1 n}\left(s_{1}\right)\right\}\right. \\
& \times\left\{f_{n}\left(z, l_{n} s_{1}\right)-f_{n}\left(-L, l_{n} s_{1}\right)\right\} l_{n} d s_{1} \\
& +i \int_{1}^{2} \gamma_{2 n}\left(s_{2}\right)\left[f_{n}\left\{z,\left(C-l_{n}\right) s_{2}+\left(2 l_{n}-C\right)\right\}\right. \\
& \left.-f_{n}\left\{-L,\left(C-l_{n}\right) s_{2}+\left(2 l_{n}-C\right)\right\}\right]\left(C-l_{n}\right) d s_{2} \\
& \left.+i \int_{C}^{\infty} \gamma_{t n}(\xi)\left\{f_{n}(z, \xi)-f_{n}(-L, \xi)\right\} d \xi\right],
\end{aligned}
$$




$$
\begin{aligned}
f_{n}(z, \xi)= & \frac{\pi}{Z_{N} h} e^{-i(\pi / 2-\beta)} \\
& \times \cot \frac{\pi}{Z_{N} h}\left\{(z-\xi) e^{-i(\pi / 2-\beta)}-\left(R_{n}+n\right) h\right\},
\end{aligned}
$$

where $s_{1}$ and $s_{2}$ are coordinates stretching with the variable cavity length $l_{n}$ and defined by

$$
\begin{aligned}
s_{1} & =\xi / l_{n} \\
& \left(0<s_{1}<1 \quad\left(0<\xi<l_{n}\right), n=0, \cdots, Z_{N}-1\right), \\
s_{2}= & \left\{\xi-\left(2 l_{n}-C\right)\right\} /\left(C-l_{n}\right) \\
& \left(1<s_{2}<2 \quad\left(l_{n}<\xi<C\right), n=0, \cdots, Z_{N}-1\right) .
\end{aligned}
$$

In the present analysis with larger inlet duct length $L(L=$ $1000 C), f(-L, \xi)$ can be approximated by $-\pi e^{i \beta} / h$. The flow around the cascade is connected to a space with a constant total pressure along $A B$ at a distance $L$ upstream from the leading edge of the blade.

The strength of singularities and the cavity length are separated into steady and unsteady components. As the disturbances are assumed to be small, the velocity $(u, v)$ is divided into the uniform steady component $(U, U \alpha)$, the steady disturbance component $\left(u_{s}, v_{s}\right)$ and the unsteady disturbance component $\left(\tilde{u} e^{j \omega t}, \tilde{v} e^{j \omega t}\right)$ as follows.

$$
\left\{\begin{array}{l}
u=U+u_{s}+\tilde{u} e^{j \omega t} \\
v=U \alpha+v_{s}+\tilde{v} e^{j \omega t}
\end{array}\right.
$$

Here, $\alpha \ll 1, U \gg\left|u_{s}\right|,\left|v_{s}\right| \gg|\tilde{u}|,|\tilde{v}|$ are assumed and linearization is made based on these assumptions throughout the present study.

Following five boundary conditions are applied to determine the flow field.

1. Pressure on the cavity surface equals to the vapor pressure.

2. Velocity component normal to wetted surface is zero.

3. Cavity thickness at cavity trailing edge is zero.

4. Pressure difference between the pressure and suction surfaces of blade vanishes at the trailing edge of the blade.

5. As a downstream duct length is assumed to be infinite, a downstream flow rate fluctuation is suppressed due to the infinite inertia effect of the fluid in the downstream duct. Thus, the fluctuation of cavity volume is related to the upstream velocity fluctuation.

The strength of singularities are specified at discrete points $s=S_{1 k}\left(k=1 \sim N_{C}\right)$ on the cavity surface and $s=S_{2 k}\left(k=1 \sim N_{B}\right)$ on the wetted surface in the coordinates stretching with the variable cavity length as unknowns. The values of $N_{C}$ and $N_{B}$ are set to be 37 as well as the previous research $^{(5)}$.

The boundary conditions are applied at the middle of those discrete points. After linearization, the boundary conditions are reduced to the following set of linear equations.

For steady components,

$$
\left[A_{s}\left(l_{s, n}\right)\right]\left\{\begin{array}{c}
q_{s}\left(S_{11}\right) / U \alpha \\
: \\
\gamma_{1}\left(S_{11}\right) / U \alpha \\
: \\
\gamma_{2}\left(S_{21}\right) / U \alpha \\
: \\
\sigma / 2 \alpha
\end{array}\right\}=\left\{B_{s}\right\}
$$

and for unsteady components,

$$
\left.A_{u}\left(l_{s, n}, \omega\right)\right]\left\{\begin{array}{c}
\tilde{q}\left(S_{11}\right) \\
: \\
\tilde{\gamma}_{1}\left(S_{11}\right) \\
\vdots \\
\tilde{\gamma}_{2}\left(S_{21}\right) \\
\vdots \\
\tilde{u}_{c, n} \\
\alpha \tilde{l}_{n}
\end{array}\right\}=\left\{\begin{array}{c} 
\\
0
\end{array}\right\}
$$

where $A_{s}\left(l_{s, n}\right)$ and $A_{u}\left(l_{s, n}, \omega\right)$ are coefficient matrices, $B_{s}$ is a constant vector. The steady flow can be determined from Eq. (7) which shows that the steady cavity length $l_{s, n}$ is a function of $\sigma /(2 \alpha)$. In this study, the cavity lengths $l_{s, n}$ are determined for a given value of $\sigma /(2 \alpha)$. By assuming the values of $l_{s, n}$, other unknowns are determined from Eq. (7) without closure conditions. The obtained values of unknowns are put into the closure conditions to check if they are satisfied. Then the corrections are made on the values of $l_{s, n}$ and the above process is repeated until the closure conditions are satisfied. Equation (8) is a set of linear homogeneous equations and it shows that the unknowns are functions of the steady cavity length $l_{s, n}$ or equivalently $\sigma /(2 \alpha)$. The determinant of Eq. (8) should satisfy the following equation so that the nontrivial solutions can be obtained:

$$
\left|A_{u}\left(l_{s, n}, \omega\right)\right|=0
$$

The complex angular frequency $\omega=\omega_{R}+j \omega_{I}$ is determined from Eq. (9). Thus, the angular frequency and the stability of flow field depend on the steady cavity length $l_{s, n}$ or equivalently $\sigma /(2 \alpha)$. The complex angular frequency is normalized by the circumferential wavelength $Z_{N} h\left(Z_{N}=4\right)$ as follows:

$$
k=k_{R}+j k_{I}=\frac{\omega /(2 \pi)}{U_{T} /\left(Z_{N} h\right)} .
$$

The real part $k_{R}$ represents the ratio of propagation velocity of disturbance to the peripheral velocity $U_{T}$ of the cascade, observed in a frame moving with the cascade. The imaginary part $k_{I}$ represents decay ratio.

\section{Results and Discussions}

Calculations were made for the cascade with alternately unequally spaced blades. Mean solidity $C / h$ is 2.0 , the number of blades $Z_{N}$ is 4 , the stagger angle $\beta$ is $80 \mathrm{deg}$ and the length $L$ of the inlet duct toward $x$-axis is $1000 C$. 


\subsection{Steady flow analysis}

The ratio of inequality $P$ in Eq. (1) was set to be 0.05 and the calculations were made for the cascade in which the spacing are $1.05 h$ between 0th (2nd) and 1st (3rd) blades and $0.95 h$ between 1st (3rd) and 2 nd (0th) blades. The cascade geometry is shown in Fig. 2. The length of the cavity on the 0th (2nd) blade in wider spacing is shown by symbols and $O$ in Fig. 2, and that on the 1st (3rd) blade in narrower spacing is shown by symbols $\boldsymbol{\Delta}$ and $\Delta$. The length of the cavity in the cascade with equally spaced blades $(P=0)$ is shown by solid and broken lines. The development process of the cavity is similar to the case of a cascade with alternate leading edge cutback $^{(2),(3)}$. In Fig. 2, $L 0$ and $L 1$ show the distances between the leading edge of the blade and the throat of blade passage.

We consider the case with decreasing $\sigma /(2 \alpha)$ from a larger value of $\sigma /(2 \alpha)$. When the value of $\sigma /(2 \alpha)$ is sufficiently large such as $\sigma /(2 \alpha)=7.0$, the length $(\boldsymbol{\Delta}$ in Fig. 2) of the cavity on the 1st (3rd) blade in wider spacing is a little larger than that on the 0th (2nd) blade in narrower spacing. As the value of $\sigma /(2 \alpha)$ decreases, the cavities on both blades develop. When the length of the cavity on the 1 st (3rd) blade in wider spacing approaches about $65 \%$ of the distance $L 0$, the cavity on the 1 st (3rd) blade starts to develop quickly and the cavity on the 0 th (2nd) blade starts to diminish.

This behavior can be explained by the interaction of the local flow with small angle of attack near the cavity closure with the leading edge of the opposing blade, which has been pointed out as the cause of the occurrence of the alternate blade cavitation and cavitation instabilities ${ }^{(5)}$. Detailed discussions will be made later.

As the value of $\sigma /(2 \alpha)$ decreases further, two couples

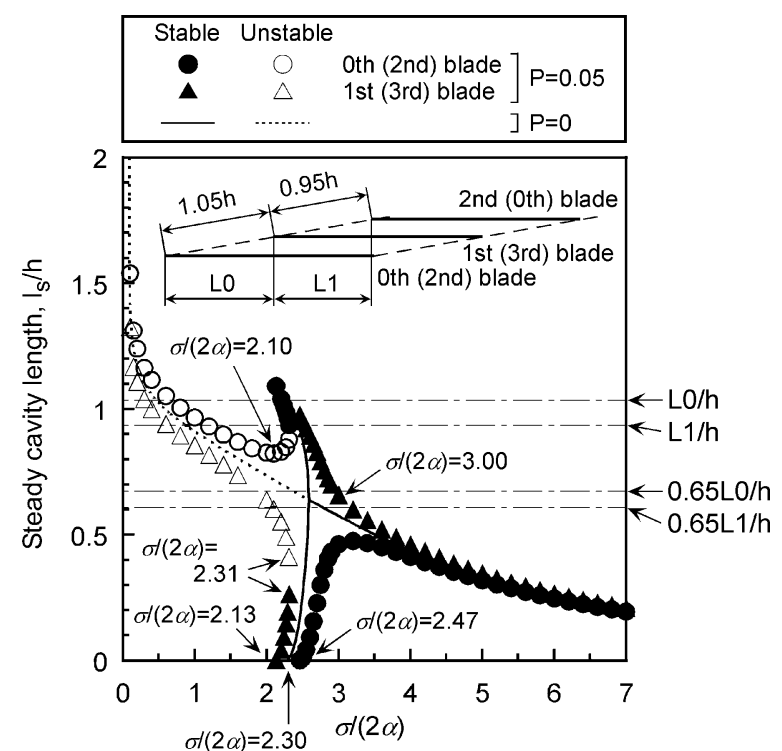

Fig. 2 Steady cavity length and its stability. $C / h=2.0, \beta=80^{\circ}$, $P=0,0.05$ of solutions can be obtained at $2.13 \leq \sigma /(2 \alpha) \leq 2.31$ after passing a region without steady solution. One is a couple of solutions shown by the symbols $\boldsymbol{\top}$ and $\boldsymbol{\Lambda}$, and the other is a couple of solutions shown by $O$ and $\Delta$. For the solution with larger difference of cavity lengths $(\boldsymbol{\bullet}, \boldsymbol{\Delta})$, the cavity on the 0th (2nd) blade develops and the cavity on the 1st (3rd) blade diminishes as the value of $\sigma /(2 \alpha)$ decreases. For the solution with smaller difference of cavity lengths $(O, \Delta)$, the cavities on all blades develop after the cavity on the 0th (2nd) blade diminishes slightly.

To clarify the relation between the flow field and the cavity development, the local angle of attack was examined on the coordinate $\xi$ along the upstream extension of 0th (2nd) and 1st (3rd) blade with the origin at the leading edge. The results are shown in Fig. 3. Bold solid and broken lines drawn vertically in Fig. 3 show the location of the trailing edge of the cavities on the 1st (3rd) and 0th (2nd) blades on the coordinate $\xi$ from the leading edges of 2nd (0th) and 1st (3rd) blades, respectively.

Figure 3 (a) shows the results for the cavitation at $0.50 \leq \sigma /(2 \alpha) \leq 7.00$ with smaller difference of cavity lengths at $2.13 \leq \sigma /(2 \alpha) \leq 2.31$. At $\sigma /(2 \alpha)=7.00$ and 5.00 , the angles of attack near the leading edge of blade $(\xi / h=-0.5 \sim 0)$ are about the same on both blades. In the region upstream of the trailing edge of the cavity, the angle of attack is larger. This is caused by the blockage effect of the cavity on the lower blade. When the values of $\sigma /(2 \alpha)$ becomes 3.00, the angle of attack near the 0th (2nd) blade leading edge becomes smaller and the cavity on the 0th (2nd) blade diminishes because the local flow with small angle of attack near the cavity closure on the 1st (3rd) blade approaches the leading edge of the 0th (2nd) blade. As the values of $\sigma /(2 \alpha)$ decreases further, the angle of attack near the leading edge of the 0th (2nd) blade becomes quite small as shown in the result for $\sigma /(2 \alpha)=2.47$ and the length of the cavity on the 0th (2nd) blade becomes about zero as shown in Fig. 2. When the cavity on the 0th (2nd) blade becomes smaller, the angle of attack near the leading edge of the 1 st (3rd) blade increases and the cavity on the 1st (3rd) blade develops. When the value of $\sigma /(2 \alpha)$ decreases from 2.47 to 2.31 , the cavity on the 0th (2nd) blade suddenly becomes longer and that on the 1 st (3rd) blade shorter.

When the value of $\sigma /(2 \alpha)$ decreases from 2.31 to 2.10 , the angle of attack near the leading edge of the 0th (2nd) blade decreases due to the development of the cavity on the opposing blade and the length of the cavity on the 0th (2nd) blade decreases slightly as shown in Fig. 2. As the value of $\sigma /(2 \alpha)$ decreases from 1.00 to 0.50 , the cavities on both blades develop and the angle of attack near the leading edge of the blade decreases. In this range of $\sigma /(2 \alpha)$, the effect of the decrease of $\sigma$ is larger than the effect of the decrease of the angle of attack and the cavity does not diminish although the angle of attack decreases. 


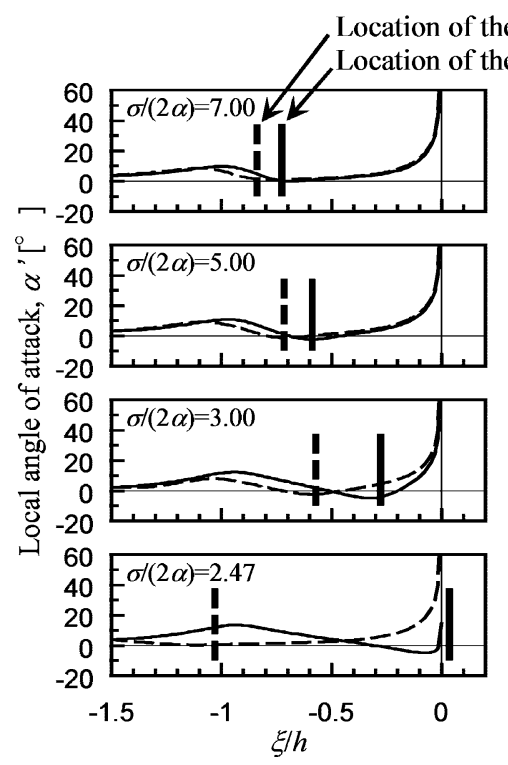

(a) For $\sigma /(2 \alpha) \geq 2.47$, and for the cavitation with smaller difference of cavity lengths for $\sigma /(2 \alpha) \leq 2.31$

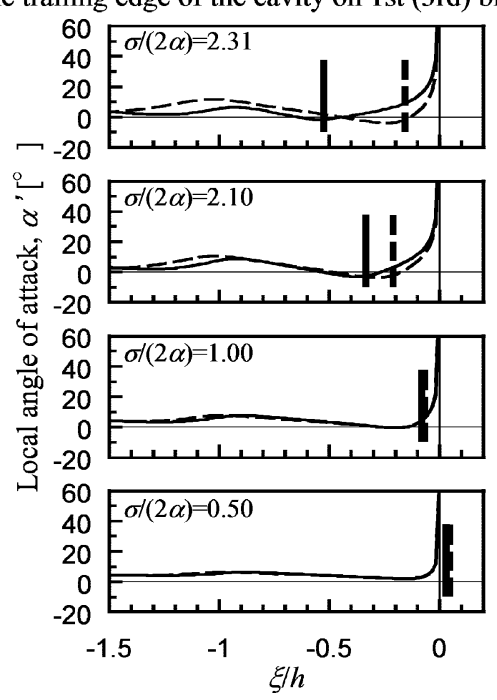

(b)

Fig. 3 Local angle of attack in the cascade with unequally spaced blades. $C / h=2.0, \beta=80^{\circ}$, $P=0.05 . \alpha=5^{\circ}$

Next, consider the case of cavitation with larger difference of cavity lengths for $2.13 \leq \sigma /(2 \alpha) \leq 2.31$, as the value of $\sigma /(2 \alpha)$ decreases, the angle of attack near the leading edge of the 1st (3rd) blade decreases due to the influence of the cavity on the opposing blade. The cavity on the 1st (3rd) blade diminishes and the cavity on the 0th (2nd) blade develops.

Thus, the development of cavity can be explained reasonably from the interaction of the local flow with small angle of attack near the cavity closure with the leading edge of the opposing blade.

In the cascade with the blades whose leading edges are alternately cut back ${ }^{(2),(3)}$, the cavity in the blade passage with longer distance between the leading edge of the blade and the throat is longer in the region of larger $\sigma /(2 \alpha)$ but shorter in the cascade with unequally spaced blade. This is the major difference from the result of alternate leading edge cutback. The present study has shown the relation between the development of the cavity and the flow field more clearly by plotting the angle of attack (Fig. 3).

\subsection{The effect of the ratio of inequality $P$ to the steady cavity}

The length of steady cavity in the cascades whose ratios of inequality $P$ are 0.10 and 0.20 are shown in Figs. 4 and 5 , respectively. The development process of the cavity is the same as that in the cascade with $P=0.05$, qualitatively.

The regions of $\sigma /(2 \alpha)$ without steady solution are $2.31 \sim 2.47$ for $P=0.05,2.13 \sim 2.65$ for $P=0.10,1.81 \sim$ 3.07 for $P=0.20$. Therefore, it is found that the region of $\sigma /(2 \alpha)$ without the steady solution increases as the value of $P$ increases.

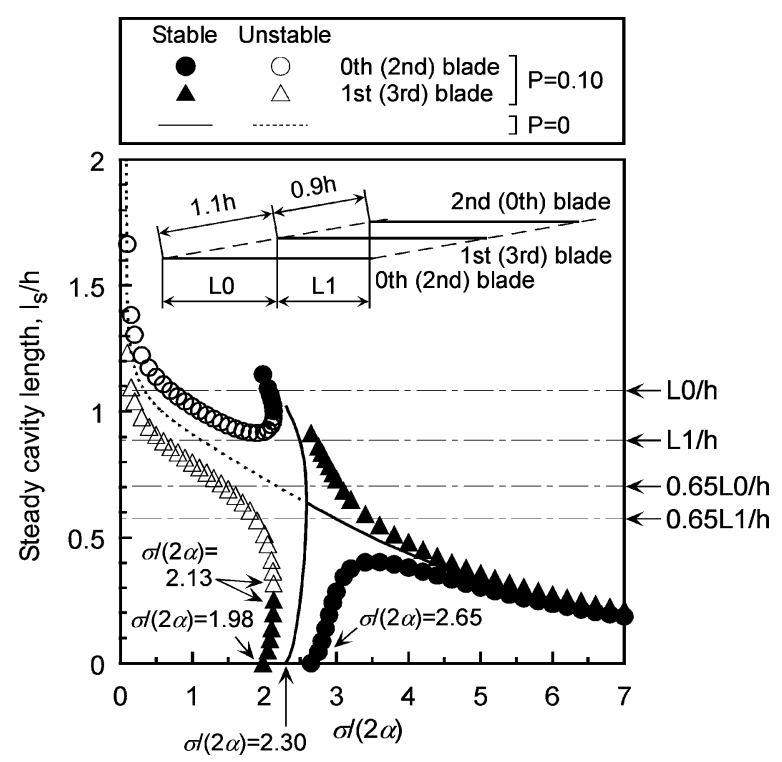

Fig. 4 Steady cavity length and its stability. $C / h=2.0, \beta=80^{\circ}$, $P=0.10$

\subsection{Stability of the cavitation in the cascade with the ratio of inequality $P=0.05$}

Figure 6 shows the reduced frequency $k_{R}$ of amplifying modes $\left(k_{I}<0\right)$ and the phase difference $\theta_{0,1}\left(=\theta_{2,3}\right)$ of the cavity length fluctuation on the 1 st ( 3 rd) blade relative to that on the 0th (2nd) blade in the case with $P=0.05$. The steady solutions examined are shown by solid lines in the upper part of each figure. Figure 6 (a) shows the results for the steady cavitation with the cavity length plotted by the solid line in the upper figure. Although many higher order modes exist, only seven modes are shown. $\theta_{n, n+2}$ in the legend represents the phase difference of the cav- 
ity length fluctuation on $(n+2)$ th blade relative to that on $n$th blade. Many unstable modes appear in the region of smaller $\sigma /(2 \alpha)$ than about 3, where the cavity is longer

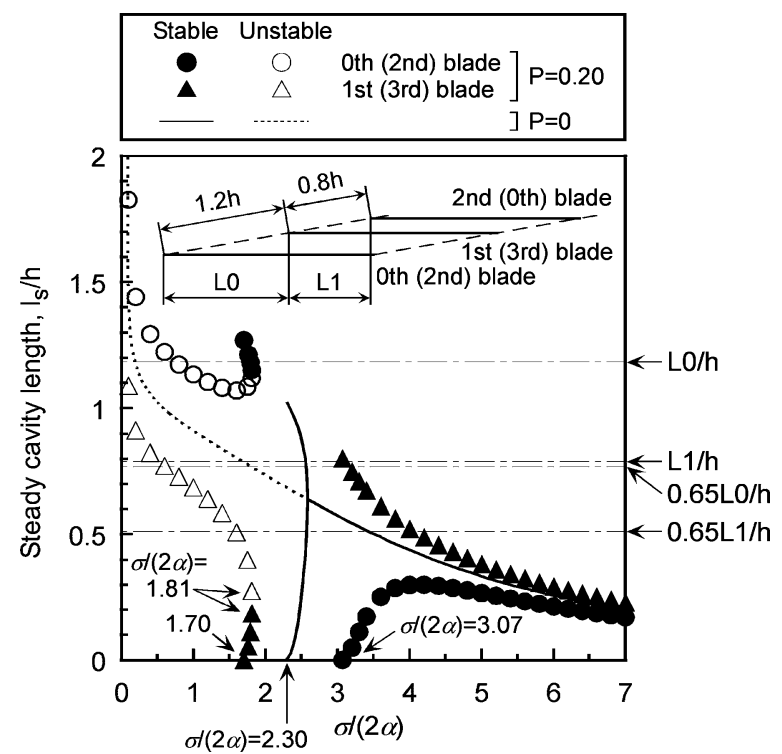

Fig. 5 Steady cavity length and its stability. $C / h=2.0, \beta=80^{\circ}$, $P=0.20$
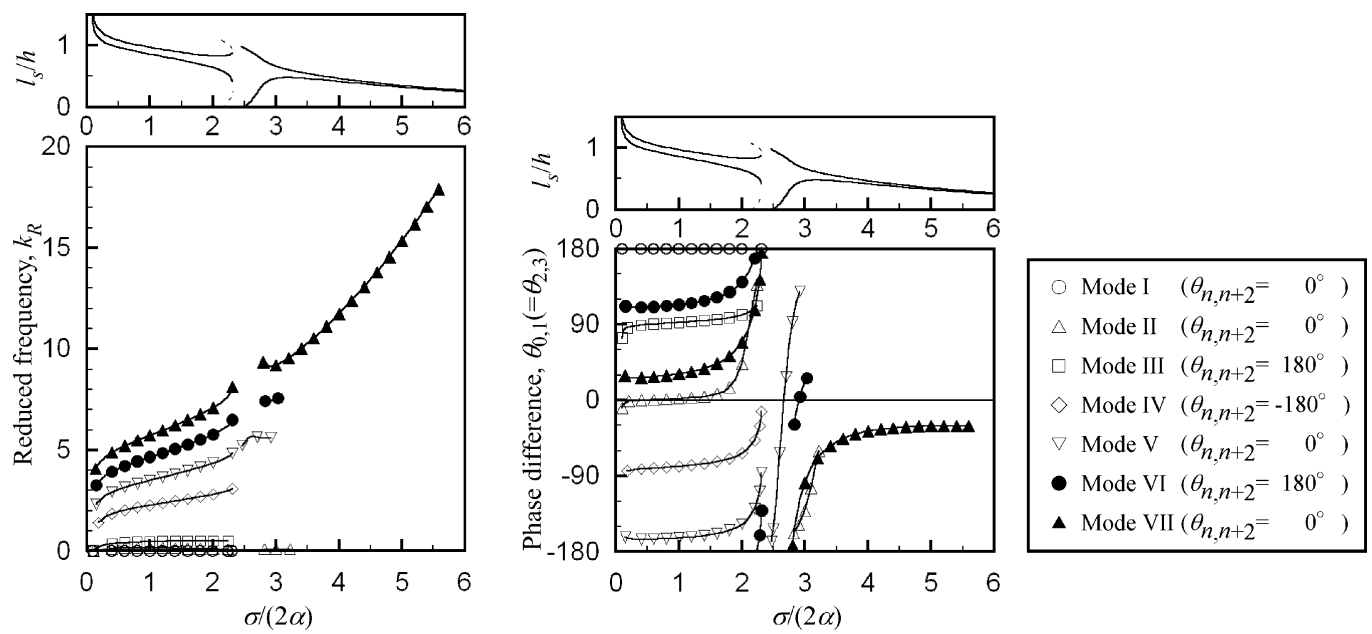

(a) For $\sigma /(2 \alpha) \geq 2.47$, and for the cavitation with smaller difference of cavity lengths for $\sigma /(2 \alpha) \leq 2.31$
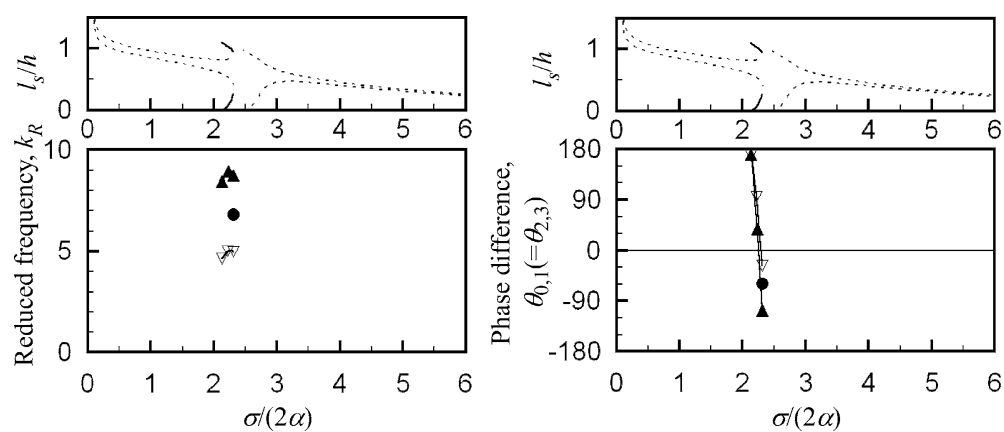

than the $65 \%$ of the distance between the leading edge of the blade and the throat.

The phase differences $\theta_{n, n+2}$ of the cavity length fluctuation in Mode I, II, V and VII are 0 deg. Mode I shown by the symbol $O$ in Fig. 6 (a) is a mode in which the frequency equals to zero and the phase angle $\theta_{0,1}\left(=\theta_{2,3}\right)$ is $180 \mathrm{deg}$. Mode I represents a statically unstable mode in which the cavity on one blade becomes longer exponentially and the cavity on the neighboring blades shorter without oscillation. Mode I appears in the region of $\sigma /(2 \alpha) \leq 2.31$. Thus, the cavitation in the region of $\sigma /(2 \alpha) \leq 2.31$ is statically unstable. It has been found ${ }^{(4)}$ that the stability of the cavitation determined from the existence of Mode I can reasonably explain experimental results. The stability of steady cavitation determined from the existence of Mode I is shown in Fig. 2.

The phase differences $\theta_{0,1}\left(=\theta_{2,3}\right)$ of Mode II, V and VII change largely as shown in the right figure of Fig. 6 (a). Mode II may correspond to the cavitation surge because $\theta_{0,1}$ is small in the region of smaller $\sigma /(2 \alpha)$. Mode $\mathrm{V}$ is thought to be the rotating cavitation with 2 cells because $\theta_{0,1}$ is $-180 \mathrm{deg}$ in the region of smaller $\sigma /(2 \alpha)$. To determine the direction of propagation, the phase of the unsteady component of velocity was examined at $\xi=-5 C$.

(b) For the cavitation with larger difference of cavity lengths for $2.13 \leq \sigma /(2 \alpha) \leq 2.31$

Fig. 6 Destabilizing roots for the cascade with unequally spaced blades. $C / h=2.0, \beta=80^{\circ}$, $P=0.05$ 
Figure 7 shows the phase difference of the unsteady component of velocity $\tilde{v}$ at the upstream $(\xi=-5 C)$ relative to that at the upstream $(\xi=-5 C)$ of 0th blade. The phase difference changes with $-720 \mathrm{deg}$ against one period $(0 \leq \zeta \leq 4 h)$ of cascade. Thus, it was confirmed that Mode $\mathrm{V}$ is the disturbance with 2 cells propagating backward (in the direction of increasing $\zeta$ ). So, Mode $\mathrm{V}$ represents a backward rotating cavitation with 2 cells. Mode VII is thought to be corresponding to the higher order mode of the cavitation surge ${ }^{(5)}$ because the phase difference is small in the region of smaller $\sigma /(2 \alpha)$.

The phase differences $\theta_{n, n+2}$ of Mode III, IV, and VI are $180 \mathrm{deg},-180 \mathrm{deg}$, and $180 \mathrm{deg}$, respectively, and rep-

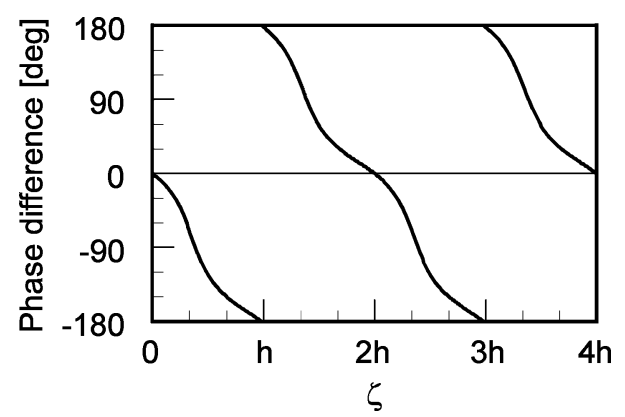

Fig. 7 Phase difference of the unsteady component of velocity $\tilde{v}$ at $\xi=-5 C$ in Mode $\mathrm{V}$ in the cascade with unequally spaced blades. $C / h=2.0, \beta=80^{\circ}, P=0.05 . \sigma /(2 \alpha)=$ 1.0 resent rotating cavitations. Mode III and VI represent the forward rotating cavitation and its higher order mode, respectively. Mode IV corresponds to the backward rotating cavitation.

Figure 6 (b) shows the reduced frequency $k_{R}$ and the phase difference $\theta_{0,1}\left(=\theta_{2,3}\right)$ of the cavity length fluctuation of the cavitation with larger difference of cavity lengths at $2.13 \leq \sigma /(2 \alpha) \leq 2.31$. Mode I with $k_{R}=0$ and $\theta_{0,1}\left(=\theta_{2,3}\right)=$ $180 \mathrm{deg}$ does not appear. Therefore, the cavitation with larger difference of cavity lengths is statically stable. The static stability of the cavitation based on the existence of Mode I is shown in Fig. 2.

Judging the stability from Mode I, it was found that the minimum value of $\sigma /(2 \alpha)$ for the stable cavitaion is 2.13, which is smaller than 2.30 for a cascade with equally spaced blades $(P=0)$.

\section{4 Stability of the cavitation in the cascade with the ratio of inequality $P=0.10$ and 0.20}

Figure 8 shows the reduced frequency $k_{R}$ of amplifying modes $\left(k_{I}<0\right)$ and the phase difference $\theta_{0,1}\left(=\theta_{2,3}\right)$ of the cavity length fluctuation for the case with $P=0.10$. The steady solutions examined are shown by solid lines in the upper part of each figure. Figure 8 (a) shows the results for the steady cavitation with the cavity length plotted by the solid line in the upper figure. Mode I appears in the region of smaller $\sigma /(2 \alpha)$ than the lower limit $(\sigma /(2 \alpha)=2.13)$ of the region without steady solution.
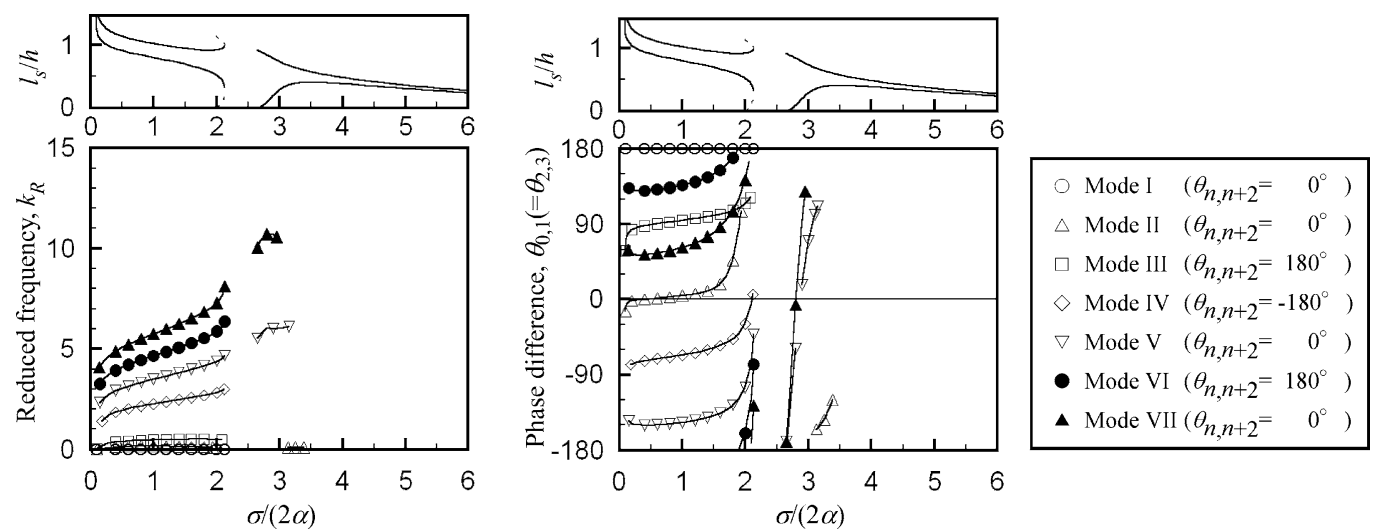

(a) For $\sigma /(2 \alpha) \geq 2.65$, and for the cavitation with smaller difference of cavity lengths for $\sigma /(2 \alpha) \leq 2.13$
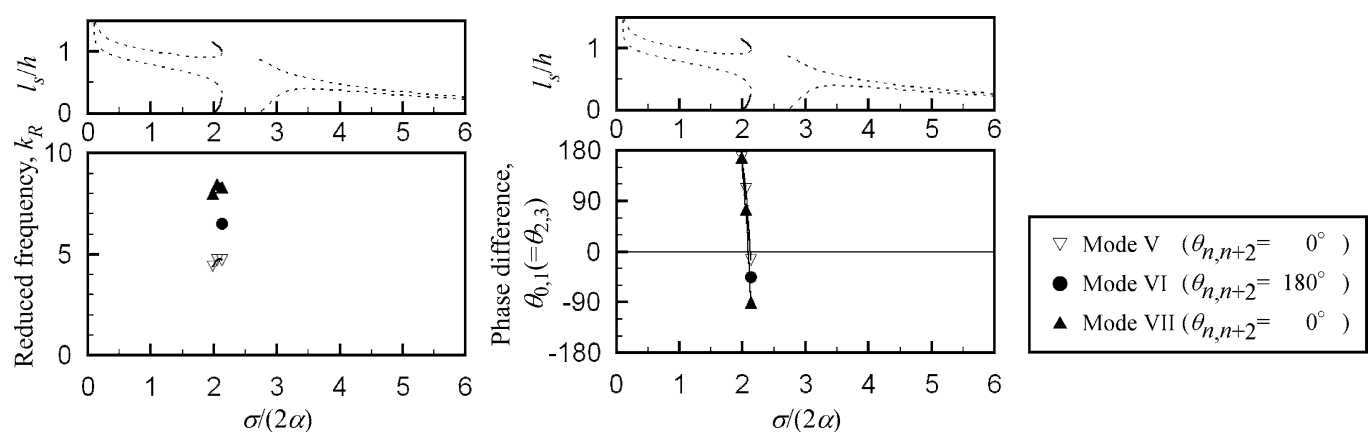

(b) For the cavitation with larger difference of cavity lengths for $1.98 \leq \sigma /(2 \alpha) \leq 2.13$

Fig. 8 Destabilizing roots for the cascade with uneven spacing. $C / h=2.0, \beta=80^{\circ}, P=0.10$ 



(a) For $\sigma /(2 \alpha) \geq 3.07$, and for the cavitation with smaller difference of cavity lengths for $\sigma /(2 \alpha) \leq 1.81$
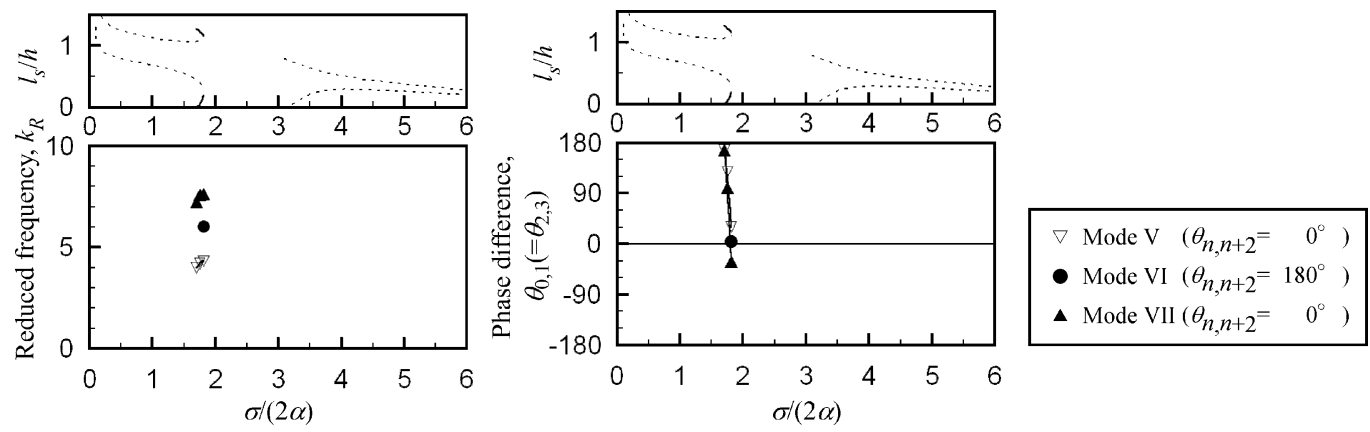

(b) For the cavitation with larger difference of cavity lengths for $1.70 \leq \sigma /(2 \alpha) \leq 1.81$

Fig. 9 Destabilizing roots for the cascade with unequally spaced blades. $C / h=2.0, \beta=80^{\circ}$, $P=0.20$

Thus, the cavitation is statically unstable in the region of $\sigma /(2 \alpha) \leq 2.13$.

In comparison with the results of the cases with $P=$ 0.05 , the region of the occurrence of Mode VII corresponding to the higher order mode of the cavitation surge is quite small.

Figure 8 (b) shows the results for the cavitation with larger difference of cavity lengths shown by the solid lines in the upper part of each figure. Mode I does not appear and the cavitation with larger difference of cavity lengths is considered to be statically stable.

The static stability of the cavitation determined from the existence of Mode I is shown in Fig. 4. The minimum value of $\sigma /(2 \alpha)$ for the stable cavitation is 1.98 , which is smaller than 2.13 for the cascade with $P=0.05$.

Figure 9 shows the reduced frequency $k_{R}$ and the phase difference $\theta_{0,1}\left(=\theta_{2,3}\right)$ of the cavity length fluctuation for the case with $P=0.20$. The steady solutions examined are shown by solid lines in the upper part of each figure. In Fig. 9 (a), Mode I appears in the region with smaller $\sigma /(2 \alpha)$ than the minimum values of $\sigma /(2 \alpha)(=1.81)$ of the region without steady solution. This is the same as for the cases of the cascade with $P=0.05$ and 0.10 . So, the cavitation is statically unstable in the region with $\sigma /(2 \alpha) \leq 1.81$.

Mode II corresponding to cavitation surge does not appear in the region with larger $\sigma /(2 \alpha)$ than the maximum values of $\sigma /(2 \alpha)(=3.07)$ of the region without steady solution. This was found for the cascade with $P=0.10$.

Figure 9 (b) shows the results for the cavitation with larger difference of cavity lengths shown by the solid lines in the upper part of each figure. Mode I does not appear and the steady cavitation with larger difference of cavity lengths is statically stable. The stability of the cavitation based on Mode I is shown in Fig. 5. The minimum value of $\sigma /(2 \alpha)$ for the stable cavitation is 1.70 .

Finally, it was found that the minimum value of $\sigma /(2 \alpha)$ for stable cavitation decreased from 2.30 for $P=0$ to 1.70 for $P=0.20$. This result suggests that the minimum value of $\sigma /(2 \alpha)$ for stable cavitation can be decreased by unequalizing the blade spacing alternately.

\subsection{Effects of the ratio of inequality $P$ on the fre- quency of the cavitation surge and the rotating cavitation}

Figure 10 shows the reduced frequency $k_{R}$ of Mode II and III corresponding to cavitation surge and rotating cavitation for different values of $P$. The frequency $k_{R}$ of Mode II increases slightly as the value of $P$ increases. The frequency of Mode III does not change except the frequency around the onset point of Mode III. It has been confirmed that the frequencies of other modes, Mode IV, V, VI and VII also are not affected by the value of $P$ except near the onset points. From these results, it was suggested that 


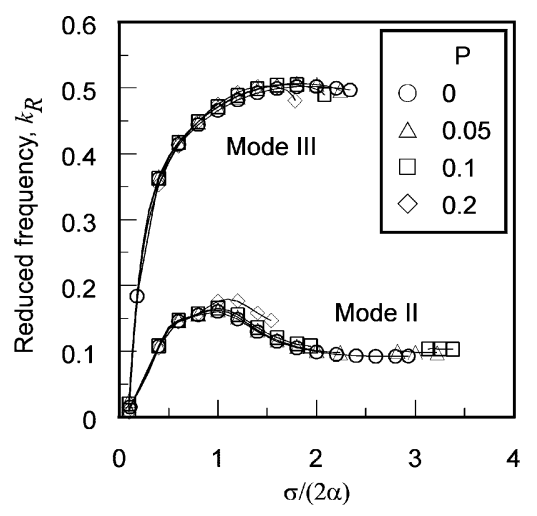

Fig. 10 Reduced frequency of Mode II and III. $C / h=2.0$, $\beta=80^{\circ}$

the frequencies of the rotating cavitation and other modes, which is usually observed, do not change by unequalizing the blade spacing alternately although the frequency of the cavitation surge increases slightly.

\section{Conclusions}

(1) For the cascades with alternately unequally spaced blades, the cavity length is a complicated function of $\sigma /(2 \alpha)$. The cavity development can be explained reasonably from the interaction of the local flow near the cavity closure with the leading edge of the opposing blade.

(2) The minimum value of $\sigma /(2 \alpha)$ with statically stable solution can be decreased by unequalizing the blade spacing alternately. The value of $\sigma /(2 \alpha)$ at the onset of cavitation instabilities such as the rotating cavitation can be also decreased by unequalizing the blade spacing. This effect is larger in the case with larger ratio of inequality. However, the region of $\sigma /(2 \alpha)$ without steady solution increases as the ratio of inequality increases.

(3) Various kinds of oscillating mode appear when the cavity becomes longer than the $65 \%$ of the distance between the leading edge of blade and the throat of blade passage, as in the case of the cascade with equally spaced blades.

( 4 ) By unequalizing the blade spacing, the frequency of the cavitation surge increases slightly and the frequencies of the rotating cavitation and other cavitation instabilities do not change except near their onset points.

\section{References}

( 1 ) Goirand, B., Mertz, A.L., Joussellin, F. and Rebattet, C., Experimental Investigations of Radial Loads Induced by Partial Cavitation with a Liquid Hydrogen Inducer, Proceedings of the Third International Conference on Cavitation (IMechE), C453/056 (1992), pp.263-269.

( 2 ) Horiguchi, H., Watanabe, S. and Tsujimoto, Y., Theoretical Analysis of Cavitation in Inducers with Unequal Blades with Alternate Leading Edge Cutback: Part I-Analytical Methods and the Results for Smaller Amount of Cutback, Trans. ASME Journal of Fluids Eng., Vol.122, No.2 (2000), pp.412-418.

( 3 ) Horiguchi, H., Watanabe, S. and Tsujimoto, Y., Theoretical Analysis of Cavitation in Inducers with Unequal Blades with Alternate Leading Edge Cutback: Part IIEffects of the Amount of Cutback, Trans. ASME Journal of Fluids Eng., Vol.122, No.2 (2000), pp.419-424.

( 4 ) Yoshida, Y., Tsujimoto, Y., Kataoka, D., Horiguchi, H. and Wahl, F., Effects of Alternate Leading Edge Cutback on Unsteady Cavitation in 4-Bladed Inducers, Trans. ASME Journal of Fluids Eng., Vol.123, No.4 (2001), pp.762-770.

( 5 ) Horiguchi, H., Watanabe, S., Tsujimoto, Y. and Aoki, M., A Theoretical Analysis of Alternate Blade Cavitation in Inducers, Trans. ASME Journal of Fluids Eng., Vol.122, No.1 (2000), pp.156-163. 